\title{
Pygopus 2: tilting the Wnt-Notch balance in mammary epithelial lineage determination
}

\author{
Kevin Roarty and Jeffrey M Rosen ${ }^{*}$
}

\begin{abstract}
The mammary gland requires the coordinated efforts of multiple epithelial cell lineages to build an organized bilayered ductal network. How epigenetic regulators direct decisions of cell-fate and lineage determination in cooperation with intrinsic transcriptional factors and extrinsic signaling factors remains a fundamental question in the field of developmental biology. Recent work sheds new light on the role of the histone methylation reader Pygopus 2 in coordinating the balance of self-renewal Wnt signals with luminalspecific Notch signals in mammary epithelial lineage determination.
\end{abstract}

The mammary gland exhibits a strikingly dynamic repertoire of morphological changes throughout the course of development, orchestrated in large part by systemic and local cues that govern developmental potential. Central to the establishment of proper tissue homeostasis, the mammary stem cell (MaSC) requires a set of lineagerestricted events in order to give rise to both progenitor and more differentiated cell types within the mammary gland. For years, a number of studies have intricately assembled individual pieces of a rather complicated puzzle, gradually providing a more complete picture of the epithelial lineage constituents and the factors orchestrating these cell fate decisions [1]. Elegant lineage tracing experiments have more recently provided valuable insight into the developmental fate of mammary stem and progenitor cells [2,3]; however, the factors that govern these outcomes remain an unresolved puzzle.

A recent study by Gu and colleagues in Cell Stem Cell [4] provides compelling evidence for the regulation of lineage determination through the histone methylation reader Pygopus 2 (Pygo2), uniquely positioned to tilt the

\footnotetext{
* Correspondence: jrosen@bcm.edu

Department of Molecular and Cellular Biology, Baylor College of Medicine, One Baylor Plaza, Houston, TX 77030-3498, USA
}

Wnt and Notch signaling balance so as to facilitate basal and luminal lineage decisions. Interactions between the Wnt and Notch pathways are well known to play important roles in cell fate determination by what has been previously coined 'Wntch' signaling [5]. The Pygopus family of proteins were originally recognized as contextdependent transcriptional coactivators of the Wnt/B-catenin signaling pathway and further shown to be capable of 'reading' the histone code, identifying and binding trimethylated lysine 4 of histone H3 (H3K4Me3) [6,7]. These histone landmarks indicate areas of active gene transcription. In the mammary gland, the epigenetic landscape depicted by both active (H3K4Me3) and repressive (H3K27me3) histone modifications was recently mapped between MaSC-enriched, luminal progenitor, and mature luminal lineages, assigning the epigenome as a key determinant of cell-fate decisions [8].

Though other chromatin players are implicated in conveying lineage decisions, the work of $\mathrm{Gu}$ and colleagues uncovered the first evidence for a chromatin reader, Pygo2, as an epigenetic modulator of cell-fate, additionally providing a functional link between two prominent signaling pathways in these decisions. Pygo2 maintains $\mathrm{MaSC} /$ basal fate specifically by suppressing luminal/alveolar differentiation, in part due to the suppression of Notch signaling and promotion of Wnt/ßcatenin signaling. What determines whether a MaSC/ basal cell turns down the path of luminal differentiation? Using a series of micro-chromatin chip assays (microChIP), the authors discovered that Pygo2 within the $\mathrm{MaSC} /$ basal epithelial cells engages $ß$-catenin to bind to the Notch3 locus and maintain Notch3 in a 'bivalent' yet repressed chromatin structure, poised and ready when the decision to undergo luminal fate specification ensues. In the MaSC, Pygo 2 and $\mathrm{B}$-catenin also bind to specific sites within the Axin2 locus. In the absence of Pygo2, the MaSC/basal cells lose their identity to some extent, adopting characteristics of a more luminal nature [4]. 
Also uncovered is a new role for Pygo2's ability as a histone code reader to act distinctly at two different loci conferring differential chromatin and transcriptional configurations with regard to Wnt and Notch pathways. In the mammary gland, these two pathways have been shown to guide MaSC self-renewal cues within the basal lineage, in the case of Wnt [3,9], and luminal lineage determination, in the case of Notch [10]. Thus, in essence, these two signaling pathways represent the foundation of their respective lineages. However, within these pathways there exists a more complicated picture beyond the broad model, where multiple cell lineages within the mammary epithelium express different ligand and receptor constituents of the Wnt and Notch families. Precisely how different luminal and basal lineages coordinate these interactions during development and how the resultant signals define lineage specification and developmental potential in vivo still remains an elusive story.

Intriguingly, there exists an inverse correlation of Pygo2 specifically with Notch3 over additional Notch1, Notch2, and Notch4 receptor paralogues [4]. Notch receptor paralogues, as well as the Notch ligands Jagged and Delta, exhibit differential expression within epithelial subpopulations [11]. Preferential Notch activity resides within the luminal compartment, while the ligands are expressed primarily in the basal compartment. Other critical modulators of this pathway, such as Lunatic fringe (Lfng), also play an important role [12]. Refinement of the epithelial constituents and differential Notch member expression suggest there is a more intricate model for Notch signaling in the luminal layer, defining lineage potential and developmental outcome. Current evidence points to Notch signaling in mediating cell-fate specification within the terminal end bud through Aurora kinase and orientation of the mitotic spindle, guiding lineage decisions when terminal end buds drive ductal extension and the formation of the epithelial bilayer [13]. Coincidentally, recent lineage tracing studies involving Notch2 and Notch3 receptors present elegant insight into the identification of previously unrecognized luminal cell populations. These studies provide a detailed understanding of the location of these cells and the fate of their progeny over time, in a sense reshaping the view of the epithelial hierarchy and the function of Notch signaling in vivo [14,15]. Lafkas and colleagues [14] recently described Notch3 expression in a clonogenic and transiently quiescent luminal progenitor population, using a conditionally inducible Notch3CreERT2 ${ }^{\mathrm{SAT}}$ transgenic mouse. Notch2 genetic fate mapping, on the other hand, labeled what the authors coined as S (Small) and L (Large) progenitors, orchestrating proper tertiary branching and alveologenesis [15]. At present, it remains to be determined how much overlap, if any, exists between these Notch2+ and Notch3+ populations.
The above studies emphasize the importance of not only the differential regulation and expression of lineage determinants, but also highlight the necessity of understanding the spatial component of where these events occur during development. Pygo2, therefore, links Wnt and Notch pathways in the specification of lineage potential. However, a critical question remains in how these pathways are then coordinated during the different phases of mammary gland development - for example, in the terminal end buds, during ductal elongation and branching throughout puberty, and upon epithelial expansion during pregnancy within milk-producing alveoli. It will take concerted efforts using multiple genetic approaches to tackle the establishment of lineage potential in vivo, guided by an understanding of cell intrinsic transcriptional regulators, extrinsic paracrine interactions, and epigenetic regulation. Clearly there is a lot still to be learned.

\section{Abbreviations}

H3K27Me3: Tri-methylated lysine 27 of histone H3; H3K4Me3: Tri-methylated lysine 4 of histone H3; MaSC, Mammary stem cell.

\section{Competing interests}

The authors declare that they have no competing interests.

\section{Acknowledgements}

JMR is supported by grant NCl-CA16303 and KR is supported by a Department of Defense Postdoctoral Fellowship W81XWH-10-1-0356.

\section{Published: 23 Dec 2013}

\section{References}

1. Visvader JE: Keeping abreast of the mammary epithelial hierarchy and breast tumorigenesis. Genes Dev 2009, 23:2563-2577.

2. Van Keymeulen A, Rocha AS, Ousset M, Beck B, Bouvencourt G, Rock J, Sharma N, Dekoninck S, Blanpain C: Distinct stem cells contribute to mammary gland development and maintenance. Nature 2013, 479:189193.

3. van Amerongen R, Bowman AN, Nusse R: Developmental stage and time dictate the fate of Wnt/beta-catenin-responsive stem cells in the mammary gland. Cell Stem Cell 2012, 11:387-400.

4. Gu B, Watanabe K, Sun P, Fallahi M, Dai X: Chromatin effector Pygo2 mediates Wnt-notch crosstalk to suppress luminal/alveolar potential of mammary stem and basal cells. Cell Stem Cell 2013, 13:48-61.

5. Hayward P, Kalmar T, Arias AM: Wnt/Notch signalling and information processing during development. Development 2008, 135:411-424.

6. Gu B, Sun P, Yuan Y, Moraes RC, Li A, Teng A, Agrawal A, Rhéaume C, Bilanchone V, Veltmaat JM, Takemaru K, Millar S, Lee EY, Lewis MT, Li B, Dai $\mathrm{X}$ : Pygo2 expands mammary progenitor cells by facilitating histone $\mathrm{H} 3$ K4 methylation. J Cell Biol 2009, 185:811-826.

7. Jessen S, Gu B, Dai X: Pygopus and the Wnt signaling pathway: a diverse set of connections. Bioessays 2008, 30:448-456.

8. Pal B, Bouras T, Shi W, Vaillant F, Sheridan JM, Fu N, Breslin K, Jiang K, Ritchie ME, Young M, Lindeman GJ, Smyth GK, Visvader JE: Global changes in the mammary epigenome are induced by hormonal cues and coordinated by Ezh2. Cell Rep 2013, 3:411-426.

9. Zeng YA, Nusse R: Wnt proteins are self-renewal factors for mammary stem cells and promote their long-term expansion in culture. Cell Stem Cell 2010, 6:568-577.

10. Robinson GW: Using notches to track mammary epithelial cell homeostasis. Cell Stem Cell 2008, 3:359-360.

11. Bouras T, Pal B, Vaillant F, Harburg G, Asselin-Labat ML, Oakes SR, Lindeman GJ, Visvader JE: Notch signaling regulates mammary stem cell function and luminal cell-fate commitment. Cell Stem Cell 2008, 3:429-441. 
12. Xu K, Usary J, Kousis PC, Prat A, Wang DY, Adams JR, Wang W, Loch AJ,

Deng T, Zhao W, Cardiff RD, Yoon K, Gaiano N, Ling V, Beyene J,

Zacksenhaus E, Gridley T, Leong WL, Guidos CJ, Perou CM, Egan SE: Lunatic fringe deficiency cooperates with the Met/Caveolin gene amplicon to induce basal-like breast cancer. Cancer Cell 2012, 21:626-641.

13. Regan JL, Sourisseau T, Soady K, Kendrick H, McCarthy A, Tang C, Brennan K, Linardopoulos S, White DE, Smalley MJ: Aurora A kinase regulates mammary epithelial cell fate by determining mitotic spindle orientation in a Notch-dependent manner. Cell Rep 2013, 4:110-123.

14. Lafkas D, Rodilla V, Huyghe M, Mourao L, Kiaris H, Fre S: Notch3 marks clonogenic mammary luminal progenitor cells in vivo. J Cell Biol 2013, 203:47-56.

15. Sale S, Lafkas D, Artavanis-Tsakonas S: Notch2 genetic fate mapping reveals two previously unrecognized mammary epithelial lineages. Nat Cell Biol 2013, 15:451-460.

$10.1186 / \mathrm{bcr} 3592$

Cite this article as: Roarty and Rosen: Pygopus 2: tilting the Wnt-Notch balance in mammary epithelial lineage determination. Breast Cancer Research 2013, 15:322 